\title{
Single Photon Transport by a Moving Atom
}

\author{
A.E. Afanasiev ${ }^{1 a}$, P.N. Melentiev ${ }^{1}$, A.A. Kuzin ${ }^{1,2}$, A.Yu. Kalatskiy ${ }^{1,2}$, and V.I. Balykin ${ }^{1}$ \\ ${ }^{1}$ Institute for Spectroscopy Russian Academy of Sciences, Moscow, Troitsk, Russia \\ ${ }^{2}$ Moscow Institute of Physics and Technology, Moscow, Russia
}

According to standard diffraction theory, apertures much smaller than the wavelength of light transmit very poorly. It follows from basic principles of wave physics that the passage of a wave through a hole that is considerably smaller than the wavelength can be neglected. In the classical work by Bethe [1] on transmission of light by a nanohole in an infinitely thin and perfectly conducting screen, a simple expression for the transmission efficiency of light has been obtained, which is scaled in relation to the hole size as $(r / \lambda)^{4}$, where $r$ is the radius of the hole, and $\lambda$ is the wavelength. Under assumptions made on the screen, the transmission efficiency falls rapidly when the wavelength becomes greater than the hole radius. Ebbesen and collaborators [2] discovered the Extraordinary Optical Transmission (EOT) through sub- $\lambda$ periodic hole array. The EOT is mediated by the aid of electromagnetic surface modes supported by the holey surfaces [3]. Here we demonstrate a new physical approach for an effective light transmission through nanohole at a single photon level. The essence of the method is the following: to excite an atom in a long-lived excite state and than to let the atom to go through the nanohole. The atom will reemit photon after its transit through the nanohole.

Schematic of single photon transport by atom is shown in Fig. 1. A beam of $\mathrm{Rb}$ atoms is directed to the sample with nanoholes. Atoms were excited into a long-lived excite state $5 \mathrm{D}_{5 / 2}$ at the transitions $5 \mathrm{~S}_{1 / 2}->5 \mathrm{P}_{3 / 2}$ and $5 \mathrm{P}_{3 / 2}>5 \mathrm{D}_{5 / 2}$. The atom excitation takes place directly next to the nanohole.

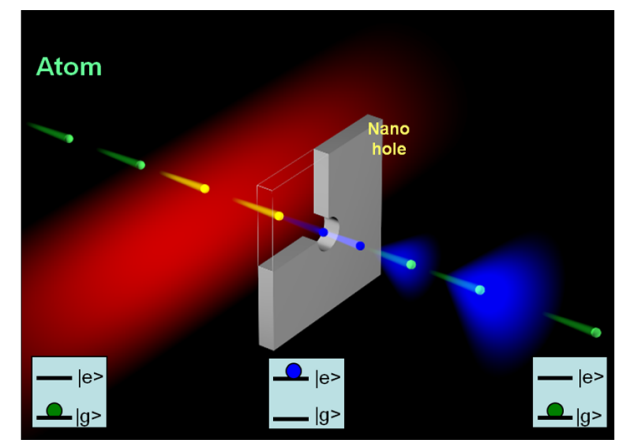

Figure 1. Scheme of single photon transport by atom through nanohole.

\footnotetext{
${ }^{\mathrm{a}}$ Corresponding author: afanasiev.isan@gmail.com
} 
As an experimental sample was used ultra-thin membrane $(40 \mathrm{~nm}, \mathrm{SiO} 2)$ with a deposited metal film on its surface. The nanoholes in a film have been created by ion beam. The diameter of nanoholes were from 175 to $540 \mathrm{~nm}$. The photon transfer efficiency through nanohole was determined by measuring the atom fluorescence after the nanohole membrane at a wavelength of $420 \mathrm{~nm}\left(6 \mathrm{P}_{3 / 2}-\right.$ $>5 \mathrm{~S}_{1 / 2}$ ). The fluorescence from an atom passed through a nanohole as a function of distance between the atom excitation region and the membrane has been investigated. Our measurements showed a high transport efficiency $1 \%$ of single photon through nanoopening for nanoholes of $400 \mathrm{~nm}$ diameter.

This work was supported by the Russian Scientific Foundation (project No. 14-12-00729).

\section{References}

1. H.A. Bethe, Phys.Rev. 66, 163 (1944)

2. T.W. Ebbesen, H.J. Lezec, H.F. Ghaemi, T. Thio, P.A. Wolff, Nature 391, 667 (1998)

3. F. J. García-Vidal, L. Martín-Moreno, T. W. Ebbesen, L. Kuipers, Rev. Mod. Phys. 82, 729 (2010) 